\title{
ADHERENCIA DE LAS ESPORAS DE Beauveria bassiana FORMULADAS EN POLVO Y LÍQUIDO SOBRE LA BROCA DEL CAFÉ
}

\section{ADHERENCY OF Beauveria bassiana SPORES FORMULATED IN POWDER AND LIQUID TO THE COFFEE BERRY BORER}

\author{
Paula F. Arrubla M. \\ Mauricio Cárdenas R. ${ }^{2}$ \\ Francisco J. Posada F. ${ }^{3}$
}

\section{RESUMEN}

El uso de trampas con alcohol, como atrayente para la captura de los adultos de la broca del café (Hypothenemus hampei (Ferrari) (Coleoptera: Curculionidae), ofrece la oportunidad de colocar depósitos de esporas del hongo Beauveria bassiana (Bals.) Vuill. (Ascomycota: Hypocreales) para la auto-inoculación y la posterior diseminación del hongo en el campo. En este estudio, se tuvo por objetivo determinar la capacidad de los adultos de la broca para recoger esporas de superficies tratadas, con cuatro métodos de aplicación y tres métodos de exposición. Las esporas, se aplicaron en polvo, suspensión en aceite [Kerosene, Carrier ${ }^{\circledR}$ y Kerosene+Carrier ${ }^{\circledR}$ (60:40)] y en diluciones de azúcar al 5 y 10\%. El mayor número de esporas recogidas, se obtuvo cuando las brocas caminaron sobre la superficie

${ }^{1}$ Bacterióloga. Universidad Católica de Manizales. Centro Nacional de Investigaciones de Café, Cenicafé, Chinchiná, Caldas, Colombia. Sede Planalto, km. 4 vía ChinchináManizales. Chinchiná (Caldas) - Colombia.

2 Diseñador Industrial. Programa jóvenes investigadores Convenio Colciencias - Cenicafé. Centro Nacional de Investigaciones de Café, Cenicafé, Chinchiná, Caldas, Colombia. Sede Planalto, km. 4 vía Chinchiná-Manizales. Chinchiná (Caldas) - Colombia.

${ }^{3}$ I. Agrónomo. Ph.D. Centro Nacional de Investigaciones de Café, Cenicafé, Chinchiná, Caldas, Colombia. Sede Planalto, km. 4 vía Chinchiná-Manizales. Chinchiná (Caldas) - Colombia. francisco.posada@cafedecolombia.com tratada con esporas en polvo. El segundo mejor método de exposición, se consiguió cuando las brocas aterrizaron sobre la superficie con las alas abiertas y el de menor adherencia, se encontró cuando las brocas aterrizaron con las alas cerradas. El tratamiento de aplicación que presentó mayor adherencia de las esporas, se obtuvo con las esporas en polvo, seguido por las formuladas en Carrier ${ }^{\circledR}$, Kerosene, Carrier ${ }^{\circledR}$, azúcar al 5 y al $10 \%$. La menor adherencia se manifestó con las esporas formuladas en Kerosene.

Palabras clave: Formulación, hongo entomopatógeno, aceite, aplicación, café

\section{SUMMARY}

Traps with alcohol, as attractant, used to capture coffee berry borer adults (Hypothenemus hampei (Ferrari) (Coleoptera: Curculionidae) give the opportunity to deposit spores of Beauveria bassiana (Bals.) Vuill. (Ascomycota: Hypocreales) to auto-inoculate the adult attracted and to get the spores disseminated into the coffee field. Therefore, the aim of this study was to evaluate the capability of the coffee berry borer adult to pick up spores, in the form of powder, from a treated surface, suspension in oil [Kerosene, Carrier ${ }^{\circledR}$ and Kerosene+Carrier $\left.{ }^{\circledR}(60: 40)\right]$ and in sugar dilution at 5 and $10 \%$. Three ways of adult exposition to the treated surfaces were evaluated. Adults walking on a surface treated with power allowed the beetles to pick up the highest number of spores. Second best expostition method was obtained when adults landed with open 
wings and the least efficient way of picking up spores was when they landed with closed wings. The most efficient formulation was spores applied as powder, followed by Carrier ${ }^{\circledR}$, Kerosene + Carrier ${ }^{\circledR}$, sugar in 5 and $10 \%$. The lowest performance was obtained when the spores were applied using Kerosene alone.

Key words: Formulation, entomopathogenic fungus, oil, application, coffee.

\section{INTRODUCCIÓN}

La broca del café Hypothenemus hampei (Coleoptera: Curculionidae), se distribuyó en los países productores de café, a partir de su sitio de origen en África y se constituyó en la plaga de mayor importancia económica a nivel mundial. Su manejo estuvo orientado por largo tiempo en el uso de insecticidas pero el desarrollo de resistencia al endosulfán y el impacto negativo de este insecticida sobre la biodiversidad y los riesgos de intoxicación, para humanos y animales promovió, a finales de 1980, el desarrollo de nuevas alternativas, como el control cultural y biológico, con un enfoque de manejo integrado (Brun et al. 1989; Bustillo et al. 1998).

Actualmente, para lograr un mayor efecto de los insecticidas y reducir su impacto ambiental negativo, existe la tendencia a disminuir su uso en la aplicación y el control de las plagas. La investigación está orientada a que su uso continúe pero apoyados en métodos que aprovechen el comportamiento de los insectos para garantizar la mayor oportunidad de establecer contacto entre el insecto y el ingrediente activo (Ford, 1996). De igual manera este enfoque se ajusta al uso de los hongos entomopatógenos que ha sido realizado convencionalmente a través de aplicaciones de formulaciones líquidas con base en agua o aceite por aspersión (Bateman, 1997; McCoy et al. 1988; Matthews, 2001) y donde se busca que las esporas se distribuyan uniformemente en las gotas asperjadas para garantizar un mayor cubrimiento de las áreas tratadas (Bateman E Alves, 2000; Matthews, 2001). Con el fin de mejorar la eficacia de los hongos, existen nuevas propuestas para su uso, empleando formulaciones en cebos (Inglis et al. 1996; Hidalgo et al. 1998; Bextine $E$ Thorvilson, 2002) o en trampas (Vega et al. 1995, Vásquez E Sánchez, 1997; Díaz \& Cotes, 1999; Klein E Lacey, 1999; Koller et al. 2005).
Estas nuevas propuestas buscan el aprovechamiento de los hábitos y el comportamiento de los insectos, para lo cual se colocan los hongos en diversos tipos de trampas, ya sea con atrayentes, como luz, cebos o feromonas. Esta práctica, se realiza para conseguir que los insectos entren en contacto con las esporas y, de este modo, obtener la exposición al inóculo y lograr la infección ya sea para obtener su control o adicionalmente para lograr la dispersión del hongo dentro del hábitat del insecto cuando éste regresa a los sitios de refugio, cría o alimentación, que comparte con otros adultos o estados inmaduros (Vega et al. 1995, Inglis et al. 1996; Vásquez E Sánchez, 1997, Hidalgo et al. 1998; Díaz \& Cotes, 1999, Smith et al. 1999; Klein E Lacey, 1999; Bextine $\varepsilon$ Thorvilson, 2002; Koller et al. 2005).

Con esta estrategia de aplicación, se busca aumentar el inóculo y mantenerlo como un factor de mortalidad en el medio. Esta es una estrategia de control de insectos con agentes biológicos denominada aumentativainoculativa, por Tanada E Kaya (1993), mientras Vega et al. (1995) proponen denominar el empleo de los insectos hospedantes para la dispersión de los hongos entomopatógenos, como auto-diseminación.

Esta documentado que los insectos transportan esporas de hongos, bien sea de patógenos de plantas o de insectos (Abbott, 2002). Con la broca del café, se demostró que los adultos son muy eficientes en la diseminación de esporas de hongos. Pérez et al. (2003) aislaron 40 especies de hongos asociados con la cutícula, intestino, heces y galerías de los frutos brocados en el campo. Dentro de estos hongos transportados por la broca, se encontró el hongo entomopatógeno Metarhizium anisopliae (Pérez et al. 2003). En el campo también se ha colectado sobre frutos de café hormigas y parasitoides que transportan esporas de $B$. bassiana, lo cual seguramente contribuye a la dispersión del hongo a los frutos atacados por la broca.

Modelos de trampas con atrayentes que permiten la autoinoculación del insecto con esporas del hongo $B$. bassiana y su posterior diseminación a los sitios de refugio han sido diseñadas y evaluadas para Carpophilus lugubris Murray (Coleoptera: Nitidulidae) (Dowd E Vega, 2003). Estos autores en los resultados demostraron que los adultos de C. lugubris, después de visitar las trampas en las cuales se colocaron los aislamientos, diseminaron el hongo a los refugios de hibernación e infectaron otros especímenes. 
Para el monitoreo de las poblaciones de la broca del café, se desarrollaron trampas para capturar adultos y las cuales utilizan como atrayente el alcohol (Mendoza, 1991; Cárdenas, 2000; Villacorta et al. 2001; Dufour, 2006). Estas trampas son los prototipos base para desarrollar nuevos diseños de trampas que permitan la atracción - inoculación - diseminación de las esporas de $B$. bassiana, por la broca del café.

Esta capacidad de los insectos para adquirir, transportar y contribuir a la diseminación de inóculo en su hábitat tiene un gran potencial para desarrollar nuevas alternativas de control utilizando trampas con atrayentes que no maten los insectos y que permitan su escape después de inocularse con esporas de los hongos entomopatógenos. Por esta razón, el primer punto para desarrollar esta tecnología de trampas para la atracción - inoculación y diseminación del hongo $B$. bassiana por la broca del café es determinar la adherencia de las esporas al cuerpo de los adultos y establecer la capacidad de adquisición de esporas cuando el insecto desarrolla actividades, como caminar y aterrizar sobre una superficie tratada con esporas en polvo y formuladas.

\section{MATERIALES Y MÉTODOS}

El experimento, se realizó, en el laboratorio de entomología de CENICAFE. Se utilizaron esporas del hongo B. bassiana (Bb9205) en polvo, producidas en la planta piloto de hongos entomopatógenos, las cuales presentaron las siguientes características: contenido de humedad $10 \%$, concentración $4,2 \times 10^{10}$ esporas/gramo, viabilidad del $95 \%$, patogenicidad $97,5 \%$, pureza $99 \%$ y un tamaño de partícula de $77 \mu \mathrm{m}$.

Se organizó el trabajo en un diseño completamente al azar con arreglo factorial $3 \times 5 \times 4$. Los tratamientos estuvieron conformados por tres métodos de exposición de las brocas a las esporas. Éstas, se aplicaron a una superficie, donde los adultos se expusieron a caminar, aterrizar con las alas abiertas y por caída sin abrir las alas. El tiempo de exposición, se cuantificó para cada adulto y se estimó en tres segundos en promedio. Los cinco métodos de aplicación de las esporas del hongo consistieron en: esporas en polvo sin formular, esporas formuladas en suspención de kerosene, en Carrier ${ }^{\circledR}$ y en la mezcla de Kerosene y Carrier ${ }^{\circledR}$, proporción 60:40, así como en dos diluciones de azúcar al 5 y al $10 \%$. Cada tratamiento tuvo cuatro réplicas y cada una contó con diez brocas. El ensayo se repitió dos veces.

La variable evaluada fue la cantidad promedio de esporas por broca que recogieron los adultos después de expuestos a cada tratamiento. Los resultados, se sometieron al análisis de varianza con un nivel de significancia del 95\% (SAS Institute, 2003).

La aplicación de las esporas, se realizó sobre cajas de Petri de vidrio (Pirex ${ }^{\circledR}$ ) de una área de $9 \mathrm{~cm}$ de diámetro. Sobre cada caja se aplicó, en polvo como en líquido, el mismo equivalente de esporas por unidad de superficie. Para el tratamiento en polvo, se aplicaron $0,002 \mathrm{~g}$ equivalente a $8,4 \times 10^{7}$ esporas. El cubrimiento deseado de las cajas de Petri, se obtuvo de la siguiente manera: para las esporas en polvo sin formular, se tomaron tres cajas de Petri, con cantidades diferentes de esporas 0,005g; $0,004 \mathrm{~g}$ y 0,002g. Las esporas, se repartieron uniformemente sobre toda la caja y, finalmente, se seleccionó la caja que contenía $0,002 \mathrm{~g}$, porque se observó la mejor distribución y cubrimiento del polvo en la superficie de la caja. Además, se buscó utilizar la mínima cantidad, con el fin de hacer el uso más eficiente de las esporas, ya que productos del hongo con alta concentración o en gran cantidad pueden resultar costosas para adelantar un programa de atracción de brocas a las trampas, con depósitos de esporas, para realizar la auto-inoculación.

Los líquidos, se aplicaron con el equipo de aspersión "Potter Spray Tower®" (Burkardscientific.co. UK). Para calibrar la cantidad de líquido, se empezó a asperjar un volumen de $0,7 \mathrm{~mL}$ hasta asegurar la descarga uniforme y sin sobrenadante sobre el fondo de la caja de Petri. Finalmente, se seleccionó el volumen de $0,2 \mathrm{~mL}$, al cual se le adicionó la cantidad de esporas $(0,002 \mathrm{~g})$. Luego de asperjadas las cajas de Petri, previamente marcadas por tratamiento, se dejaron secar por un tiempo de 48 horas, a temperatura ambiente $\left(22 \pm 3^{\circ} \mathrm{C}\right)$, para formar una película delgada o matriz que retuviera las esporas. Esta aplicación debía permitir exponer los adultos de la broca caminando y aterrizando sobre la superficie tratada y garantizar que los adultos quedaran en condiciones de no adherirse a las superficies o quedar saturados de esporas.

Las brocas utilizadas, se obtuvieron de la unidad de cría de CENICAFÉ y consistieron de adultos recién 
emergidos de los frutos. Antes de iniciar el experimento los adultos, se desinfectaron con hipoclorito de sodio al $0,5 \%$ durante diez minutos y luego se lavaron tres veces con agua destilada estéril sobre mallas de tul esterilizadas (Vélez et al. 1997). Con la ayuda de un pincel, las brocas se colocaron sobre una toalla de papel estéril y se seleccionaron las más activas para ser lanzadas en vuelo, utilizando un equipo de lanzamiento que permitió que las brocas volaran y pudieran aterrizar en las superficies tratadas, simulando la forma de contacto que hace la broca en campo cuando llega a los árboles de café. La broca aterriza sobre hojas, ramas o frutos con las alas extendidas y, posteriormente, camina o choca, contra estas estructuras, con las cerradas, por lo que rebota y necesita volver a emprender vuelo (Figura 1) (Posada, 1998).

En las evaluaciones, se simularon éstas formas de la broca de entrar en contacto con el árbol del café y se expusieron a las superficies tratadas con esporas. Una vez las brocas entraron en contacto con la superficie, se tomaron rápidamente, en un tiempo menor de tres segundos, con un pincel y se colocaron diez brocas por vial con $1 \mathrm{~mL}$ de agua destilada estéril o kerosene (cuatro viales por tratamiento). El recuento de esporas, se hizo utilizando la cámara de Neubauer (Vélez et al. 1997) y la cantidad de esporas se estimó como el promedio de esporas por broca.

\section{RESULTADOS Y DISCUSIÓN}

En los diferentes tratamientos durante el tiempo de exposición de los adultos no se observó adherencia de brocas a las superficies tratadas o sobresaturación de esporas adheridas al cuerpo de las brocas. Igualmente, no se registró germinación de las esporas.

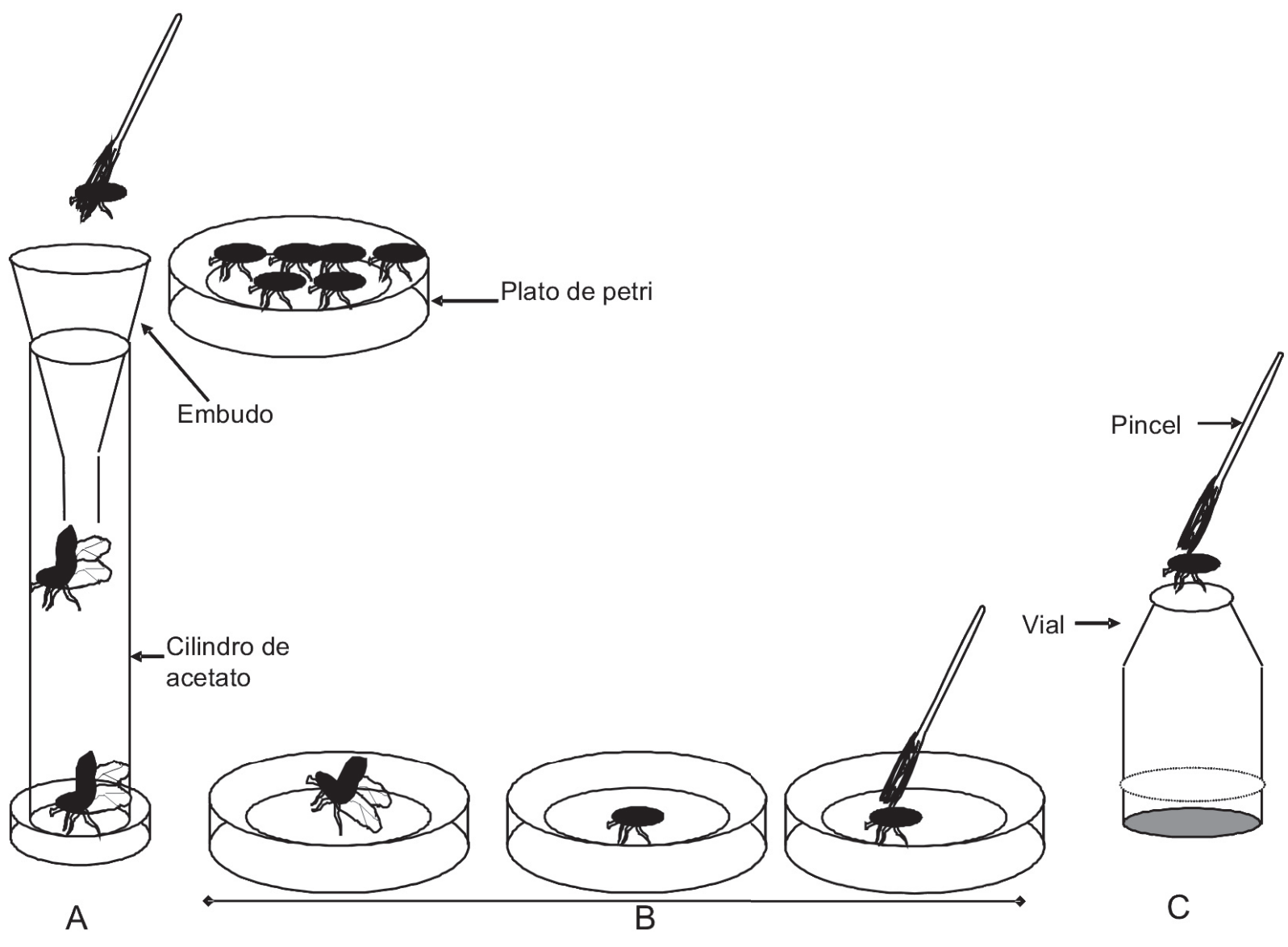

Figura 1. Equipos y procedimientos utilizados en el lanzamiento de las brocas del café para exponerlas a las esporas del hongo $B$. bassiana: $A$. Plataforma de lanzamiento; B. Exposición de las brocas a las esporas y C. Colocación de las brocas en frascos viales con la ayuda de un pincel para proceder a la cuantificación de las esporas (Posada, 1998). 
En la figura 2, se presentan los resultados de la adherencia de las esporas al cuerpo de la broca del café cuando ésta camina y aterriza sobre una superficie tratada con esporas aplicadas en polvo y formuladas en líquido. La mayor adherencia de las esporas al cuerpo de la broca, se encontró con las esporas sin formular aplicadas en polvo y cuando los adultos caminaron sobre la superficie tratada con esporas del hongo.
El método de exposición que permitió la mayor adherencia de las esporas al cuerpo de la broca, se presentó cuando los adultos se expusieron caminando, seguido por las brocas aterrizando con las alas abiertas y la menor adherencia cuando las brocas aterrizaron con las alas cerradas.

El método de exposición que permitió obtener el mayor número de esporas recogidas por la broca fue cuando los

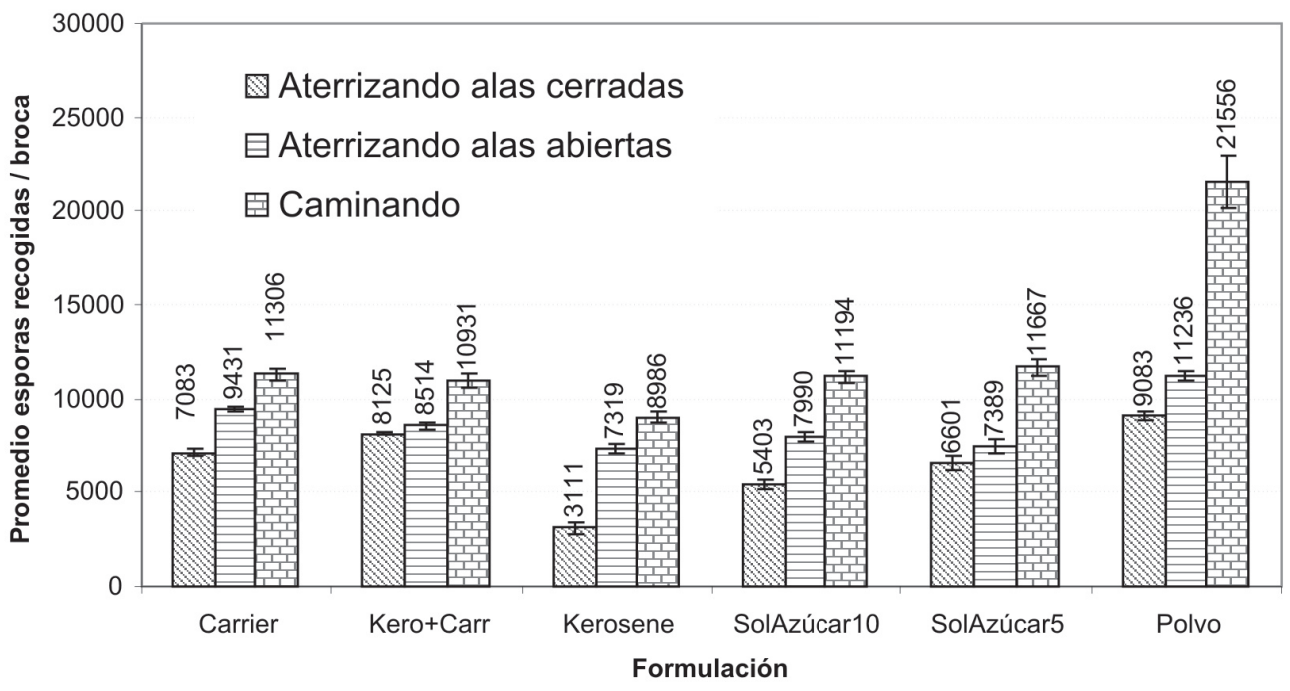

Figura 2. Promedio de esporas recogidas por la broca del café de una superficie tratada con esporas en suspensión y en polvo las barras representan la desviación estándar).

adultos caminaron sobre las esporas en polvo $(21,556$ esporas/broca), seguido del tratamiento en el cual los adultos aterrizaron con las alas abiertas sobre las esporas aplicadas en la dilución de azúcar al 5\% (11,236 esporas/ broca) y, finalmente, la menor cantidad de esporas la recogieron los adultos que aterrizaron con las alas cerradas sobre la superficie asperjada con la formulación en kerosene (3,111 esporas/broca) (Figura 2).

El número promedio de esporas recogidas por la broca presentó diferencias significativas para la interacción de los factores principales, la formulación y el método de exposición ( $\mathrm{GL}=10,84, \mathrm{~F}=23,6 ; \mathrm{P}=0.0001)$. Igualmente, se halló diferencia significativa cuando se evaluaron independientemente, los factores simples, formulación (GL $=10,84, \mathrm{~F}=23,6 ; \mathrm{P}=0.0001$ y el método de exposición $(\mathrm{GL}=2,84, \mathrm{~F}=336,9 ; \mathrm{P}=0.0001)$.

En cuanto a las formulaciones, las esporas en polvo presentaron la mayor adherencia a los adultos de la broca, seguido por las formulaciones en Carrier $^{\circledR}$ y en Kerosene+Carrier ${ }^{\circledR}$, luego las formulaciones preparadas en diluciones de azúcar al 5\% y al 10\%. La menor efectividad, se obtuvo cuando solo se utilizó kerosene (Figura 2).

El resultado de la mayor adherencia de las esporas al cuerpo de la broca cuando los adultos se expusieron a las esporas en polvo y caminando, probablemente, se explica porque las esporas en polvo estaban sueltas o libres. Es posible que en esta condición, las esporas se encuentren más disponibles a adherirse a la cutícula del insecto, ya que ésta tiene la misma constitución hidrofóbica de las esporas de $B$. bassiana, lo que facilita el contacto entre espora e insecto cuando los adultos de la broca desarrollan actividad sobre los depósitos de esporas (Wagner E Lewis, 2000).

Igualmente, la abundancia de setas que cubren el cuerpo de la broca puede ser otro factor que contribuye 
a incrementar el área superficial y, por tanto, aumenta la posibilidad de la adquisición de las esporas cuando éstas se encuentran aplicadas en forma de polvo sobre una superficie y los adultos desarrollan la actividad de caminar o aterrizar.

La mayor adquisición de esporas por la broca, cuando la exposición de los adultos se realizó caminando, se pudo deber a que el cuerpo de la broca se encontró en contacto directo con las esporas en la superficie tratada. En ese tratamiento, aunque el tiempo de exposición fue corto, los adultos alcanzaron a desplazarse caminando sobre la superficie, lo que pudo contribuir a que recogieran la mayor cantidad de esporas en comparación con los métodos de aterrizar, donde los insectos se recogieron inmediatamente después de realizar contacto con la superficie tratada. La broca es un insecto que después de aterrizar sobre las hojas, ramas y frutos del árbol del café gasta muy poco tiempo para empezar a penetrar en los frutos (Posada, 1998).

Estos resultados donde la broca recoge una gran cantidad de esporas en un corto tiempo, tanto caminando o aterrizando con las alas abiertas o cerradas, son de gran importancia para orientar los trabajos de investigación que buscan entender cómo ocurre el encuentro del insecto con las aplicación de las esporas de los hongos entomopatógenos. Adicionalmente, estos resultados permiten considerar el desarrollo de nuevas metodologías de aplicación para lograr que sea más eficiente el uso de los hongos entomopatógenos en el control de insectos plagas.

Respecto a las formulaciones preparadas con los aceites y lasdiluciones de azúcar, la menor adherencia a la broca probablemente se debió a que las esporas estaban retenidas por las formulaciones a la superficie tratada como a una matriz. En esta condición, la transferencia de las esporas exige mayor fuerza para transferirse y adherirse al cuerpo del insecto. La retención de las esporas por la formulación no permite la rápida transferencia de las esporas cuando la broca está desarrollando la actividad de caminar y de aterrizar, como ocurre cuando las esporas están libres (polvo). Esta forma de aplicación puede ofrecer la oportunidad de dosificar la transferencia de las esporas al cuerpo de la broca. Además, puede garantizar la mayor permanencia de las esporas en las trampas y ampliar la frecuencia de renovación de los depósitos, lo cual conlleva a reducir costos.
El siguiente estimativo entre las esporas recogidas por la broca y la concentración letal 50 (CL50) establecida en estudios previos (Posada, 1998) confirma que en todos los métodos de exposición y de formulación, los adultos de la broca adquieren esporas suficientes para lograr su infección y de diseminación en el campo. Si se tiene en cuenta la menor cantidad de esporas adheridas que se obtuvo con la aplicación kerosene y las brocas aterrizando con las alas cerradas, la cifra de esporas recogidas representa 1556 y 135 veces, respectivamente, la $\mathrm{CL}_{50}$ de 2 y 23 esporas $/ \mathrm{cm}^{2}$, determinada para brocas expuestas a superficies asperjadas con esporas formuladas en Kerosene +Carrier ${ }^{\circledR}$ y Carrier ${ }^{\circledR}+$ agua (Posada, 1998). Estos datos indican que la autoinoculación y la diseminación de las esporas del hongo $B$. bassiana por la broca es muy factible que ocurra y lo que se requiere es el diseño de las trampas que permitan la atracción y el escape de las brocas infectadas para que regresen al campo, como ha sido demostrado para otros insectos en los trabajos de Klein E Lacey (1999), Dowd E Vega (2003) y Koller et al. (2005).

Para propósitos prácticos de diseño de trampas y de aplicar en el campo las esporas en estos dispositivos, cebados con atrayentes para la broca del café, en las cuales los adultos se impregnan con las esporas, se debe tener en cuenta que las esporas aplicadas en polvo no van a permanecer por más largo tiempo que las formuladas en aceite y en diluciones de azúcar, debido a que las esporas en polvo pueden ser removidas con mayor facilidad por el viento (García E Ignoffo, 1977).

La retención de las esporas en las matrices formadas por el aceite o las diluciones de azúcar tienen la ventaja de evitar la remoción por el viento y de garantizar la permanencia de las esporas por más tiempo en los depósitos, así la transferencia de las esporas al insecto sea baja comparada con las aplicadas en polvo. Estas características de retención son promisorias en proyectos de uso de trampas con atrayentes y el uso de esporas para la auto-inoculación, diseminación e infección de insectos en el campo. Probablemente, este tipo de aplicación contribuya a hacer más eficiente el uso de los recursos y a disminuir las labores de cambio de depósitos de inóculo si el uso de trampas con depósitos de esporas, se llega a establecer como un sistema de aplicación para el control de la broca del café. 
En estos estudios del uso de trampas con atrayentes para la auto-inoculación y diseminación de esporas utilizando las adultos hembras de la broca, se espera que éstas regresen a los árboles atacados e infecten con el inóculo a otras adultos cuando llegan a los frutos brocados o inoculen los frutos visitados cuando transportan las esporas. Una broca puede visitar varios frutos en un corto periodo de tiempo después de aterrizar (Posada, 1998). En frutos brocados, donde ya hay brocas dentro de las perforaciones, la nueva broca explora pero no penetra y, generalmente, prefiere hacer su propia perforación, por lo que bajo estas condiciones, se puede observar frutos hasta con diez orificios de penetración, con actividad de brocas y muchas de ellas colonizadas por el hongo $B$. bassiana (Posada, 1998).

Estos resultados de la adherencia de las esporas al cuerpo de la broca del café abren nuevas posibilidades de investigación donde se debe buscar establecer cuál es el grado infección sobre la población de la broca y establecer por cuánto tiempo es posible mantener las esporas aplicadas en las trampas. Adicionalmente, se debe determinar la infección y la diseminación del hongo a través de las brocas. En este nueva propuesta de uso del hongo es necesario investigar cuál es el efecto de los factores ambientales sobre la capacidad de germinación y patogenicidad de las esporas colocadas en las trampas, con el objetivo de poder planear las actividades que conlleven el uso de trampas con depósitos de formulaciones del hongo y donde los resultados garanticen que se convierta en un método de aplicación que contribuya al control efectivo de la broca del café.

El desarrollo de trampas para la diseminación de esporas del hongo B. bassiana es una investigación que se debe continuar y recibir apoyo. Puede llegar a ser una herramienta para la introducción temprana del hongo en el agroecosistema cafetero sincronizando las etapas de desarrollo de los frutos de café con los vuelos que indican la emergencia y el tránsito de los adultos (Cárdenas, 2000; Dufour, 2006; Posada et al. 2003). Con esta intervención, se lograría la introducción temprana de inóculo del hongo utilizando la misma broca para la transmisión horizontal, obtener la infección de las brocas y la producción de nuevas esporas, con lo que se puede estimular la aparición de temprana de epizotias sobre la población de la broca.

\section{CONCLUSIONES}

El experimento demostró que en la actividad de vuelo y aterrizaje de la broca del café, sobre una superficie tratada con esporas y en un corto tiempo de exposición presentó transferencia de esporas al cuerpo de los adultos que seguramente contribuyen a la auto-inoculación, a causar su infección y a la diseminación de las esporas a otros sitios dentro del cultivo de café.

Los resultados de la forma de aplicar las esporas en las formulaciones demostraron que se puede optimizar la cantidad de esporas que las brocas pueden recoger buscando garantizar la infección y mantener por mayor tiempo disponibles las esporas sobre las superficies tratadas.

En todos los tratamientos, la cantidad de esporas recogidas por los adultos se considera suficiente para garantizar la infección y para diseminar las esporas en el campo después que visitan y escapan vivas de las trampas.

\section{AGRADECIMIENTOS}

Los autores expresan sus agradecimientos a CENICAFÉ por financiación y recursos para realizar esta investigación y al auxiliar de investigación Eduardo Osorio de la disciplina de Entomología de CENICAFÉ, por la colaboración en la ejecución de los experimentos. A dos revisores anónimos y a Juan Carlos López por las observaciones y correcciones que contribuyeron a mejorar esta publicación.

\section{BIBLIOGRAFÍA}

ABBOTT, S.P. 2002. Insects and other arthropods as agents of vector-dispersal in fungi. 5p. Disponible desde Internet en: www. Thermapure.com/pdf/Abbottinsectdispersal.pdf (con acceso 06/18/07).

BATEMAN, R. 1997. Methods of application of microbial pesticide formulations for the control of grasshoppers and locusts. Memoirs of the Entomological Society of Canada pp. 69-81. En: Goettel, M.; Johnson, D.L. (Eds.), Microbial Control of Grasshoppers and Locusts. Memoirs of the Entomol. Soc. Canada. 171p. 
BATEMAN, R.P.; ALVES, R.T. 2000. Delivery systems for mycoinsecticides using oil-based formulations. Aspects of Appl. Biol. 57:163-170.

BEXTINE, B.; THORVILSON, H.G. 2002. Field Applications of bait-formulated Beauveria bassiana alginate pellets for biological control of the red imported fire ant (Hymenoptera: Formicidae). Environ. Entomol. 31(4):746-752.

BRUN, L.O.; MARCILLAUD, C.; GAUDICHON, V.; SUCKLING, D.M., 1989, Endosulfan resistance in Hypothenemus hampei in New Caledonia, J. Econ. Entomol. 82:1311-1316.

BUSTILLO, A.E; CÁRDENAS, R.; VILLALBA, D.A.; BENAVIDES, P.; OROZCO, J.; POSADA, F.J. 1998. Manejo integrado de la broca del café Hypothenemus hampei (Ferrari) en Colombia. Cenicafé, $134 \mathrm{p}$.

CÁRDENAS M., R. 2000. Trampas y atrayentes para monitoreo de poblaciones de broca del café $\mathrm{Hy}$ pothenemus hampei (Ferrari) (Col., Scolytidae). En: Memorias Simposio Latinoamericano de Caficultura, 19. San José (Costa Rica), Octubre 2-6, 2000. ICAFE-PROMECAFE, p.369-379.

DÍAZ, M.A; COTES, A.M. 1999. Desarrollo de un preformulado a base de Beauveria bassiana para su aplicación en trampas. Resúmenes XXXVI Congreso Soc. Col. Entomol. 143p.

DOWD, P.F.; VEGA, F.E. 2003 Autodissemination of Beauveria bassiana by sap beetles (Coleoptera: Nitidulidae) to overwintering sites. Biocontrol Sci. Techn. 13(1):65-75.

DUFOUR, B.P. 2006. Elaboración de un método estándar para la evaluación del trampeo de la broca del café Hypothenemus hampei Ferr. Bol. Promecafé (Costa Rica). 109:5-9.

FORD, M.G. 1996. Impact of formulation properties on insecticidal efficacy. Brighton Crop Protection Conference - Pests and Diseases. 2:801-806.

GARCÍA, C.; IGNOFFO, C.M. 1977. Dislodgment of conidia of Nomuraea rileyi form cadavers of cab- bage looper, Trichoplusia ni. J. Invertebr. Path. 30:114-116.

HIDALGO, E.; MOORE, D.; LE PATOUREL, G. 1998. The effect of different formulation of Beauveria bassiana on Sitophilus zeamais in stored maize. J. Stored Prod. Res. 34(2-3):171-179.

INGLIS, G.D.; JOHNSON, D.L.; GOETTEL. M.S. 1996. An oil-bait bioassay method used to test the efficacy of Beauveria bassiana against grasshoppers. J. Invertebr. Pathol. 67:312-315.

KLEIN, M.G.; LACEY, L.A. 1999. An attractant trap for autodissemination of entomopathogenic fungi into population of the Japanese bettle Popillia japonica (Coleoptera: Scarabaeidae). Biocontrol Sci. Techn. 9(2):151-158.

KOLLER, R.; JUNG, K.; SCHEU, S.; ZIMMERMANN, G.; RUTHER, J. 2005. Biocontrol of the forest cockchafer (Melolontha hippocastani): Experiments on the applicability of the "Catch and Infect"Technique using a combination of attractant traps with the entomopathogenic fungus Beauveria brongniartii.IOBC/wprs Bull. 28(2):37-44.

MATTHEWS, G. 2001. Can biological agents be sprayed like chemical pesticides? Pesticide Outlook - April 2001. p.60-61.

McCOY, C.W.; SAMSON; R.A.; BOUCIAS, D.G. 1988. Entomogenous fungi. En: Ignoffo, C.M. (Ed.), Handbook of Natural Pesticides, Vol. 5, Microbial Insecticides, Part A, Entomopathogenous Protozoa and Fungi. CRC Press, Boca Raton, Florida. p.151-236.

MENDOZA M., J.R. 1991. Resposta da broca-do-café, Hypothenemus hampei, a estímulos visuais e semioquímicos. Viçosa: UFV,. (Thesis - M.Sc.). 44p.

PÉREZ, J.; INFANTE, F.; VEGA, F.E. HOLGUÍN, F.; MACÍAS, J.; VALLE, J.; NIETO, G.; PETERSON, S.W.; KURTZMAN, C.P.; O'DONNELL, K. 2003. Mycobiota associated with the coffee berry borer (Hypothenemus hampei) in Mexico. Mycol. Res. 107:879-887. 
POSADA F., F.J. 1998. Production, formulation and application of Beauveria bassiana for control of Hypothenemus hampei in Colombia. University of London. (England). Ph.D. Dissertation. 227p.

POSADA F., F.J.; BUSTILLO P., A.E.; JIMÉNEZ Q., M. 2003. Seguimiento y captura de brocas usando trampas en cafetales. Brocarta (Colombia) 35:12.

SAS, INSTITUTE. 2003. SAS/Stat. User's guide. SAS Institute Inc., Cary, NC. 1028p.

SMITH, S.M.; MOORE, D.; KARANJA, L.W.; CHANDI, E.A. 1999. Formulation of vegetable fat pellets with pheromone and Beauveria bassiana to control the larger grain borer, Prostephanus truncatus (Horn.). Pesticide Sci. 55(7):711-718.

TANADA, Y.; KAYA, H.K. 1993. Insect pathology. Academic Press, Inc., San Diego, California. 666p.

VÁSQUEZ A.; N.C.; SÁNCHEZ, G.G. 1997. Bioecología y manejo de las chizas (Coleoptera: Melolonthidae) en el sistema de reproducción de arracacha en el municipio de Cajamarca, Tolima. En: Sociedad Colombiana de Entomología. Memorias III Seminario regional de control biológico. Ibague, Mayo 16 de 1997. sp.
VEGA, F.E.; DOWD, P.F.; BARTELT, R.J. 1995. Dissemination of microbial agents using an autoinoculating device and several insect species as vectors. Biol. Control. 5(4):545-552.

VÉLEZ A., P.E.; POSADA F., F.J.; MARÍN M., P.; GONZÁLEZ G., M.T.; OSORIO V., E.; BUSTILLO P., A.E. 1997. Técnicas para el control de calidad de formulaciones de hongos entomopatógenos. Bol. Técnico Cenicafé 17:1-34.

VILLACORTA, A.; POSSAGNOLO, A.F.; SILVA, R.Z.; RODRIGUES, P.S. 2001. Um modelo de armadilha com semioquímicos para o manejo integrado da broca do café Hypothenemus hampei (Ferrari) no Paraná. In: Simpósio brasileiro de pesquisa dos cafés do brasil, 2, Vitória, 2001. Proceedings Brasília: Embrapa Café, p.2093-2098.

WAGNER, B.; LEWIS, L.C. 2000. Colonization of corn, Zea mays, by the entomopathogenic fungus Beauveria bassiana. Applied Environm. Microbiol. 66(8):3468-3473.

Recibido: Agosto 24 de 2007

Aceptado: Febrero 29 de 2008 